\title{
THE TRANSMISSION OF HEPATITIS VIRUS BY ROUTINE IMMUNIZATION PROCEDURES
}

\author{
By F. P. ELLIS, O.B.E., M.D., M.R.C.P.*
}

There is ample evidence that any of the usual inoculation procedures introduces a risk of conveying infection from one subject to another unless a separate sterile syringe and needle are used for each inoculation (Bigger, 1943; Salaman, King, Williams \& Nicol, 1944; Sheehan, 1944; Hughes, 1946; Evans \& Spooner, 1950; W.H.O. Expert Committee, 1953).

Yet it is not easy to incriminate any one immunization procedure as a vector of hepatitis virus. Acquired immunity may be high, and obscure what might be otherwise obvious infections. The incubation period may be prolonged. There are often other equally likely paths of infection to confuse the issue, such as direct contact with a case of hepatitis, arsenotherapy, diagnostic venepuncture, immunizations against other infections or various types of parenteral therapy.

During 1943, at a naval establishment, thirty out of eighty-one men $(37 \%)$ admitted with 'infectious hepatitis' developed the illness within 2 months of the dates on which they were inoculated against the typhoid and para-typhoid groups of infections (Ellis, 1953a). This proportion was significantly greater than would have been expected if there had been no association between the time of onset of hepatitis and the date of inoculation. In order to amplify the information obtained in this survey the Admiralty requested all naval medical officers in charge of patients to report certain details for each person in the Royal Navy who acquired non-surgical jaundice between 1 June 1944 and 9 January 1948.

\section{METHODS}

The information requested was as follows:

(1) Name.

Number.

Rank or rating.

Ship or establishment.

Source of notification (if not as above).

Years of pre-war naval service.

Age.

Date of joining for present war.

Grog or teetotal.

(2) Clinical history, including date of onset of:

(i) Illness.

(ii) Jaundice.

(iii) Previous illness in a 'relapsed case'.

(3) Previous jaundice (dates).

* Surgeon Commander, Royal_Navy. 
(4) Previous contact with jaundice cases (dates).

(5) Number of jaundice cases in ship in previous 6 months.

(6) Blood, plasma or serum transfusions, or anti-serum injections (dates, amounts, batch numbers).

(7) Details of syphilitic infection. Type of syphilis. Initial and current Wasserman reaction or Kahn test. Date of first W.R.

(8) Details of syphilitic treatment, designation (trade name), and total amounts of arsenic and bismuth preparations. Dates of first and last injections of each. Method of syringe sterilization.

(9) Previous sulphanilamide therapy. (Drug, amount given and dates.)

(10) Relevant medical history. Malaria, dysentery, typhoid, burns, severe illness or operation, etc. (dates).

(11) Exposure to organic chemicals (T.N.T., $\mathrm{CCl}_{4}$ ).

(12) Last routine inoculation or vaccination (dates) with:

(i) Yellow fever vaccine (batch number).

(ii) T.A.B. or T.A.B.T.

(iii) Tetanus toxoid (alone).

(iv) Vaccinia vaccine.

(v) Cholera vaccine.

(13) Alcohol consumption (nil, moderate or heavy).

The returns which gave the dates of onset of either the illness or jaundice and also the dates of inoculation against typhoid and para-typhoid or of vaccination against smallpox were selected for further examination. As there were too few women to permit a reliable separate statistical examination to be made, the men and women were included together. The selected reports were then re-sorted to exclude those (1) who had neither been vaccinated nor inoculated within the 360 days preceding the date of onset of illness, (2) who had been vaccinated against yellow fever or inoculated against plague, ${ }^{*}$ cholera or tetanus (separately from T.A.B.) within the 12 months preceding the onset of illness, (3) who had been in contact with another person who was jaundiced within 90 days of the onset of illness, (4) who had received blood, plasma or serum transfusions or anti-serum injections, (5) who had been under investigation or treatment for syphilis, (6) who had received sulphanilamide treatment for gonorrhoea, as many in this category would have been subjected to diagnostic venepuncture to exclude a concurrent syphilitic infection, and (7) 'relapsed cases'. When cases were reported by two or more authorities, for example, by the medical officer of a ship and the medical officer-in-charge of the hospital to which the man was admitted subsequently, and perhaps by the naval medical officer of health for the area, the duplicated reports were rejected.

The purpose of this investigation was to determine whether the numbers, referred to in the remainder of the returns, of those who had been inoculated against typhoid or vaccinated against smallpox within the probable incubation periods for hepatitis before they developed symptoms, were greater than would be expected if there was no relationship between the onset of symptoms and these

* This information was quite often included although it was not requested. 
immunization procedures. The World Health Organization's Expert Committee (1953) recognizes the incubation periods to lie between 15 and 40 days for infectious hepatitis and between 60 and 160 days for serum or inoculation hepatitis. The numbers who had either been inoculated or vaccinated in each 30-day period between the 11th and 340th days preceding the onset of illness were therefore determined, together with the numbers which would be 'expected' if equal numbers of the naval community at risk were immunized during each 30-day period and on the hypothesis that the incidence of hepatitis was not influenced by either immunization procedure. A $\chi^{2}$ test was applied to show whether the 'reported' numbers were significantly greater or less than the 'expected' numbers.

\section{RESULTS}

Altogether 4105 returns were received for men and women serving in the Royal Navy during these years who had contracted non-surgical jaundice. Of these, 716 returns-633 for men and 83 for women (V.A.D. or W.R.N.S.)-contained recorded dates of onset of illness and the dates of either inoculation, or vaccination or of both, and showed that within the 12 months preceding illness the person in question had not experienced any other form of parenteral immunization or therapy such as arsenotherapy or blood transfusion and was not a known contact.

Table 1. Numbers of men and women who had been vaccinated or inoculated between the 11 th and 340th days preceding the onset of hepatitis, shown for 30-day periods

(The numbers 'expected' in each period are calculated on the assumption that equal numbers of the nava community were immunized during each 30-day period and the hypothesis that the incidence of hepatitis was no influenced by the immunization procedure.)

Days preceding onset of hepatitis

\begin{tabular}{|c|c|c|c|c|c|c|c|c|c|c|c|c|c|}
\hline & No's & $\begin{array}{c}11- \\
40\end{array}$ & $\begin{array}{c}41- \\
70\end{array}$ & $\begin{array}{l}71- \\
100\end{array}$ & $\begin{array}{c}101- \\
130\end{array}$ & $\begin{array}{c}131- \\
160\end{array}$ & $\begin{array}{c}161- \\
190\end{array}$ & $\begin{array}{c}191- \\
220\end{array}$ & $\begin{array}{c}221- \\
250\end{array}$ & $\begin{array}{c}251 \\
280\end{array}$ & $\begin{array}{c}281- \\
310\end{array}$ & $\begin{array}{c}311- \\
340\end{array}$ & $\begin{array}{l}11- \\
340\end{array}$ \\
\hline Period & expected & \multicolumn{12}{|c|}{ Numbers vaccinated against smallpox } \\
\hline 1944 & $13 \cdot 2$ & 15 & 9 & 7 & 17 & 14 & 17 & 13 & 19 & 16 & 12 & $6^{*}$ & 145 \\
\hline 1945 & $17 \cdot 1$ & 22 & 15 & 23 & 10 & 13 & 18 & 14 & 19 & 18 & 15 & 21 & 188 \\
\hline $1946-7$ & $4 \cdot 2$ & 8 & 3 & 2 & 6 & 5 & 4 & 3 & 3 & 4 & 3 & 5 & 46 \\
\hline \multirow[t]{2}{*}{$1944-7$} & $34 \cdot 5$ & 45 & 27 & 32 & 33 & 32 & 39 & 30 & 41 & 38 & 30 & 32 & 379 \\
\hline & & \multicolumn{12}{|c|}{ Numbers inoculated against typhoid } \\
\hline 1944 & $16 \cdot 7$ & $26^{*}$ & 18 & 16 & 17 & 14 & 18 & 16 & 13 & 20 & 14 & 12 & 184 \\
\hline 1945 & $27 \cdot 9$ & $44 * *$ & $44^{* *}$ & 29 & 24 & 31 & 26 & $17^{*}$ & 29 & 23 & 19 & 21 & 307 \\
\hline $1946-7$ & $7 \cdot 7$ & 9 & 6 & 7 & 7 & 8 & 7 & 6 & 9 & 7 & 7 & 12 & 85 \\
\hline $194 \pm-7$ & $52 \cdot 4$ & $79^{* * *}$ & $68^{*}$ & 52 & 48 & 53 & 51 & 39 & 51 & 50 & 40 & 45 & 576 \\
\hline
\end{tabular}

*, **,*** indicate that the numbers 'reported' differ significantly from the numbers 'expected' at the 5,1 or $0.1 \%$ levels.

The total numbers available were 379 who had been vaccinated and 576 who had been inoculated between the 11th and 340th days preceding the onset of the symptoms of hepatitis.

In Table 1 it is shown that for each of the war years 1944 and 1945 the numbers who were inoculated against typhoid between the 11 th and 40th days preceding the onset of illness were significantly greater than the numbers to be expected if there was no relationship between these two events; and, for the total period under review-1944 to 1947 inclusive - this difference was highly significant. This was 
not so for the two post-war years 1946 and 1947 ; but the numbers available then for analysis were very small. During 1945 the observed numbers were also greater than the expected numbers for persons who were inoculated between the 41st and 70th days before the onset of illness.

The reported numbers did not differ from the expected numbers for any of the 30-day periods for persons who had been vaccinated within 6 months of the onset of illness.

\section{DISCUSSION}

As the incubation period is said to lie between 15 and 40 days for virus $A$ of infectious hepatitis, and between 60 and 160 days for virus $B$ of serum or inoculation hepatitis, it is clear that, if some of those who developed the illness within 10-40 days of being inoculated against typhoid were infected by this injection, the virus infecting them would have been virus $\mathrm{A}$.

It is quite reasonable to suppose that virus $A$, as well as virus B, may be transmitted by injections, for both kinds of disease have been produced experimentally by inoculation of blood; indeed, the two viruses may be close variants of a single virus. Infectious hepatitis occurred commonly in the wartime naval community. and the diffuse distribution of cases in a single command is well shown by the hospital admissions from different Portsmouth establishments to the Royal Naval Hospital at Haslar during the first 4 years of the war (Ellis 1953b). This, coupled with the coming and going which is inseparable from life in the Navy particularly in wartime, and frequent mass-immunization campaigns, provided favourable conditions for the transmission of virus $\mathrm{A}$, if the sterilization or inoculation techniques permitted this to occur.

The figures used were scrutinized carefully to exclude factors which might have biased the analysis in favour of the period between the 11th and 40th days before the onset of illness, but none was discerned.

Ninety per cent of the total returns (4105) related to ratings and $10 \%$ to officers, which corresponds, closely, to the proportions of ratings serving. The officer and rating incidence of hepatitis was approximately the same during these years. Most of the selected returns $(96 \%)$ related to naval ratings who would normally carry their pay books containing the dates of their last inoculation and vaccination with them: 95 out of 100 naval rating patients who were questioned at random recently in a naval hospital produced their pay books at once. There is little doubt that the dates on the returns which were used can be relied on in the great majority of cases.

The date of the 'last' T.A.B. injection was asked for. Thus men who had received two injections would have had two opportunities to become infected in this way, but only if the virus were transmitted during inoculation.

In view of the emphasis which has been placed recently on the danger of transmitting hepatitis virus during transfusion, it is of interest to record that in this series illness occurred within 180 days of transfusion in only 27 out of 4105 cases $(0.66 \%)$. None had been transfused within 40 days of the onset of illness and the dates were not available for two others.

In support of the hypothesis that some infections were transmitted during inoculation, there are the strong theoretical considerations and experimental 
evidence which show how cross-infections might occur; the large measure of agreement with the findings of the 1943 survey, in which cases were not selected; and the fact that the figures for vaccinated persons do not indicate that infections occurred during this procedure, although the data were treated in exactly the same way as those for inoculated persons. Despite the observation by Smith (1950) that men may become infected with hepatitis virus when tattooed with inadequately sterilized instruments, skin scarification during vaccination does not seem to cause such infections in the Royal Navy.

Since it seems evident that T.A.B. inoculations carry a small risk of producing hepatitis, the technique of inoculation should be designed to ensure that such infections do not occur. Attention is drawn to the advice of the W.H.O. Expert Committee on Hepatitis, which embodies full details of the precautions which ought to be taken.

\section{SUMMARY}

The returns relating to naval personnel who were reported to the Admiralty to have acquired non-surgical jaundice between 1944 and 1947 were examined.

Where the dates of onset of illness and of inoculation were available, the numbers who developed the illness within 11-40 days of inoculation were greater than the numbers to be expected if there was no association between the dates of onset of illness and the dates of inoculation. During 1945 the observed numbers were also greater than the expected numbers when 41-70 days intervened between inoculation and the illness.

The interpretation and practical implications of the findings are discussed.

This paper is published by kind permission of the Medical Director General of the Navy, Surgeon Vice-Admiral Sir Alexander Ingleby-Mackenzie.

I am indebted to Dr F. O. MacCallum for his continued interest and for invaluable suggestions; to Dr J. O. Irwin for scrutinizing the script; to Miss H. M. Ferres who applied the statistical tests to the figures in Table 1; to Surgeon Commander M. A. Rugg-Gunn, Royal Navy, for the information about the ratings' pay books, and to Senior Commissioned Wardmaster J. L. T. Burns, Royal Navy, and Sick Berth Petty Officer (L) D. E. Lee, Royal Navy, who tabulated the numerical data.

BTGGER, J. (1943). Lancet, i, 457.

\section{REFERENCES}

Ellis, F. P. (1953a). J. Hyg., Camb., 51, 145.

Eulis, F. P. (1953b). J.R. nav. med. Serv. 39, 63.

Evans, R. J. \& Spooner, E. T. C. (1950). Brit. med. J. ii, 185.

Hughes, R. R. (1946). Brit. med. J. ii, 685.

Salaman, M. H., King, A. J., Williams, D. I. \& Nicor, C. S. (1944). Lancet, ii, 7.

SheEhan, H. L. (1944). Lancet, ii, 8.

SMrTh, B. F. (1950). J. Amer. med. Soc. 144, 1074.

World Health Organization's Expert Commitee on Hepatitis (1953). Tech. Rep. Wld Hlth Org. no. 62. 